\title{
MONOTONICITY IN TERMS OF ORDER OF THE ZEROS OF THE DERIVATIVES OF BESSEL FUNCTIONS
}

\author{
LEE LORCH
}

(Communicated by Kenneth R. Meyer)

\begin{abstract}
An elementary Sturm technique is shown to provide an alternative and simpler proof of the result that the known monotonicity of the zeros of fixed rank of the Bessel function of the first kind implies monotonicity for the zeros of its derivative for orders between -1 and 0 . The reasoning applies to other Bessel functions.
\end{abstract}

The chief objective is to show that Sturm methods suffice to infer the monotonicity in $\nu,-1<\nu<0$, of $j_{\nu k}^{\prime}$ from that of $j_{\nu k}, k=1,2, \ldots$.

Only minor changes are required to show more directly than in [3] but also by Sturm methods that $j_{\nu k}^{\prime}$ increases when $\nu>0$ and so this is recorded as well. For the same reason a result for the Bessel function of the second kind, $Y_{\nu}(x)_{\nu}$, $\nu>-1 / 2$, is also noted. Similar remarks apply to other Bessel functions.

There are Sturm-type proofs for the monotonicity of $j_{\nu k}, \nu>0,[1,3]$ and also for $j_{\nu k}^{\prime}, \nu>0,[3]$ but none such have been offered for $-1<\nu<0$. For this interval, monotonicity has been established otherwise both for $j_{\nu k}[2 ; 6$, p. 509] and for $j_{\nu k}^{\prime}$ [4].

As usual, $j_{\nu k}$ and $j_{\nu k}^{\prime}$ denote the respective $k$-th positive zeros of $J_{\nu}(x)$ and its derivative, $k=1,2, \ldots$, except that $j_{01}^{\prime}=0$ to assure continuity as $\nu \rightarrow 0+$. This is the Bessel function of first kind and order $\nu$. It satisfies the differential equation.

$$
\left(x y_{\nu}^{\prime}\right)^{\prime}+x^{-1}\left(x^{2}-\nu^{2}\right) y_{\nu}=0, \quad y_{\nu}=J_{\nu}(x) .
$$

Use will be made of the function

$$
W(x)=J_{\mu}(x)\left[x J_{\nu}^{\prime}(x)\right]-J_{\nu}(x)\left[x J_{\mu}^{\prime}(x)\right]
$$

and its derivative which, in view of (1), can be expressed as

$$
x W^{\prime}(x)=\left(\nu^{2}-\mu^{2}\right) J_{\nu}(x) J_{\mu}(x) .
$$

Received by the editors January 26, 1989 .

1980 Mathematics Subject Classification (1985 Revision). Primary 33A40, 34C10.

Supported by the Natural Sciences and Engineering Research Council of Canada. 
Required also are the inequalities

$$
j_{\nu k}<j_{\mu, k+1}, \quad-1<\mu<\nu \leq \mu+1, \quad k=1,2, \ldots .
$$

These follow from the interlacing relations in [6, p. 479] since $j_{\nu k} \leq j_{\mu+1, k}$. Theorem A [4]. For each fixed $k=1,2,3, \ldots$, the function $j_{\nu k}^{\prime}$ increases for $-1<\nu<0$.

Proof. It is required to establish that

$$
j_{\mu k}^{\prime}<j_{\nu k}^{\prime}, \quad-1<\mu<\nu<0, \quad k=1,2, \ldots
$$

Here $\mu^{2}>\nu^{2}$. Hence

$$
W^{\prime}(x)<0, \quad j_{\nu k}<x<j_{\mu, k+1} .
$$

Moreover, $W\left(j_{\mu, k+1}\right)=-j_{\mu, k+1} J_{\nu}\left(j_{\mu, k+1}\right) J_{\mu}^{\prime}\left(j_{\mu, k+1}\right)>0$.

Therefore, $W(x)>0, j_{\nu k}<x<j_{\nu, k+1}$.

If $j_{\nu k}^{\prime} \geq j_{\mu, k+1}$, the conclusion would be obvious. In case $j_{\nu k}<j_{\nu k}^{\prime}<j_{\mu, k+1}$, then $W\left(j_{\nu k}^{\prime}\right)>0$. Thus,

$$
0<-j_{\nu k}^{\prime} J_{\nu}\left(j_{\nu k}^{\prime}\right) J_{\mu}^{\prime}\left(j_{\nu k}^{\prime}\right)
$$

so that $(-1)^{k+1} J_{\mu}^{\prime}\left(j_{\nu k}^{\prime}\right)>0$, since $(-1)^{k} J_{\nu}\left(j_{\nu k}^{\prime}\right)>0$, for $-1<\nu<0$.

This establishes that $j_{\nu k}^{\prime}>j_{\mu k}^{\prime}$, as asserted.

Theorem B [5, p. 248; 6, p. 510]. For each fixed $k=1,2,3, \ldots$, the function $j_{\nu k}^{\prime}$ increases, $\nu>0$.

Proof. With $\nu>\mu>0$, it will be shown that $j_{\nu k}^{\prime}>j_{\mu k}^{\prime}$. Without restricting generality, $\nu$ may be taken less than $\mu+1$, so that (2) holds. Here $\nu^{2}>\mu^{2}$ and so

$$
W^{\prime}(x)>0, \quad j_{\nu, k-1}<x<j_{\mu k}, \quad k=1,2, \ldots,
$$

where $j_{\nu 0}=0$ so as to include the case $j_{\nu 1}^{\prime}$; here $J_{\nu}(0)=J_{\mu}(0)=0$.

Now, $W\left(j_{\nu, k-1}\right)=j_{\nu, k-1} J_{\mu}\left(j_{\nu, k-1}\right) J_{\nu}^{\prime}(j \nu, k-1) \geq 0, k=1,2, \ldots$ Hence, $W(x)>0, j_{\nu, k-1}<x<j_{\mu k}$. For $j_{\nu k}^{\prime} \geq j_{\mu k}$ the conclusion is obvious. For $j_{\nu, k-1}<j_{\nu k}^{\prime}<j_{\mu k}$, it follows that $W\left(j_{\nu k}^{\prime}\right)>0$, whence $J_{\nu}\left(j_{\nu k}^{\prime}\right) J_{\mu}^{\prime}\left(j_{\nu k}^{\prime}\right)<0$. Thus, $(-1)^{k+1} J_{\mu}^{\prime}\left(j_{\nu k}^{\prime}\right)>0$ so that $j_{\nu k}^{\prime}>j_{\mu k}^{\prime}$, as asserted.

Remark. The same line of reasoning allows one to infer that $y_{\nu k}^{\prime}$ increases, $\nu>-1 / 2$, from the known result [6, p. 509] that $y_{\nu k}$ increases for $\nu>-1 / 2$, for fixed $k=1,2, \ldots$. As usual $y_{\nu k}, y_{\nu k}^{\prime}$ denote the respective $k$ th positive zeros of the Bessel function $Y_{\nu}(x)$ and its derivative.

In carrying out the details, it must be remembered both that $y_{\mu k}<y_{\nu k}<$ $y_{\mu+1, k},-1 / 2<\mu<\nu$, with $\nu<\mu+1$, and that

$$
y_{\mu+1, k}<y_{\mu, k+1}, \quad \mu>-\frac{1}{2}, \quad k=1,2, \ldots,
$$

as is implicit in [6, §15.22, pp. 479-480]. 


\section{REFERENCES}

1. M. Bôcher, On certain methods of Sturm and their applications to the roots of Bessel's functions, Bull. Amer. Math. Soc. 3 (1897), 205-213.

2. M. E. H. Ismail and M. E. Muldoon, On the variation with respect to a parameter of zeros of Bessel and q-Bessel functions, J. Math. Anal. and Appl. 135 (1988), 187-207.

3. L. Lorch, Elementary comparison techniques for certain classes of Sturm-Liouville equations, in Proc. Uppsala 1977 Inter. Conf. Diff. Equations, Symposia Univ. Upsaliensis Annum Quingentesimum Celebrantis 7, Acta Univ. Upsaliensis, Uppsala 1977, 125-133.

4. L. Lorch and P. Szego, On the zeros of derivatives of Bessel functions, SIAM J. Math. Anal. 19 (1988), 1450-1454.

5. F. W. J. Olver, Asymptotics and special functions, Academic Press, New York and London, 1974.

6. G. N. Watson, A treatise on the theory of Bessel functions, 2nd ed., The University Press, Cambridge, 1952.

Department of Mathematics, York University, North York, Ontario, M3J IP3 Canada 